\title{
On the expansion of the resolvent for elliptic boundary contact problems
}

\author{
Thomas Krainer
}

\begin{abstract}
Let $A$ be an elliptic operator on a compact manifold with boundary $\bar{M}$, and let $\wp: \partial \bar{M} \rightarrow Y$ be a covering map, where $Y$ is a closed manifold. Let $A_{C}$ be a realization of $A$ subject to a coupling condition $C$ that is elliptic with parameter in the sector $\Lambda$. By a coupling condition we mean a nonlocal boundary condition that respects the covering structure of the boundary.

We prove that the resolvent trace $\operatorname{Tr}_{L^{2}}\left(A_{C}-\lambda\right)^{-N}$ for $N$ sufficiently large has a complete asymptotic expansion as $|\lambda| \rightarrow \infty, \lambda \in \Lambda$. In particular, the heat trace $\operatorname{Tr}_{L^{2}} e^{-t A_{C}}$ has a complete asymptotic expansion as $t \rightarrow 0^{+}$, and the $\zeta$-function has a meromorphic extension to $\mathbb{C}$.
\end{abstract}

\section{Introduction}

This paper deals with the pursuit of Seeley's program [13, 14 for elliptic operators on singular spaces that are given by a compact smooth manifold $\bar{M}$ with boundary together with a prescribed gluing rule that identifies finitely many boundary points with each other. The spaces under consideration include, in particular, quantum graphs $(\underline{\mathbf{6}}, \mathbf{7}])$ and $\mathbb{Z} / k$-manifolds $([\mathbf{1}, \mathbf{9}])$.

More precisely, following [10, we assume that the boundary of $\bar{M}$ is equipped with a covering $\wp: \partial \bar{M} \rightarrow Y$. The base manifold $Y$ is closed, and we do not assume that it is connected. In particular, $\wp$ may have a different number of sheets over each connected component of $Y$. The singular space $M_{\text {sing }}$ is obtained by collapsing the fibres $\wp^{-1}\{y\}$ to $y$ for every $y \in Y$.

A quantum graph represents a one-dimensional example of such a space: $\bar{M}$ is a disjoint union of intervals - the edges of the graph - and $Y$ is the set of vertices. For $y \in Y$, the set $\wp^{-1}\{y\}$ consists of those endpoints of edges that are joined to form the vertex $y$.

Another example for the situation under study is a disjoint union $\bar{M}=\bar{N}_{1} \cup \bar{N}_{2}$ of smooth compact manifolds $\bar{N}_{j}$ with the same (or diffeomorphic) boundary $Y=$ $\partial \bar{N}_{j}, j=1,2$. We get a 2 -sheeted covering $\wp: \partial \bar{M} \rightarrow Y$, and by collapsing the points in $\wp^{-1}\{y\}$ to $y$ for every $y \in Y$ the manifolds $\bar{N}_{j}$ are glued along their common boundary to give a closed manifold. This is the setup for surgery in

2000 Mathematics Subject Classification. Primary: 58J32; Secondary: 58J35, 35J40, 35P05.

Key words and phrases. Boundary and transmission problems, transfer and contact problems, heat equation method, $\mathbb{Z} / k$-manifolds, quantum graphs. 
spectral theory, and $\bar{M}$ is a particular example for a $\mathbb{Z} / 2$-manifold. The resulting space $M_{\text {sing }}$ is nonsingular in this situation.

The elliptic operators to be considered on $\bar{M}$ are subject to boundary conditions that respect the coupling of boundary points given by $\wp$. We will refer to these conditions as coupling conditions in the sequel (they are called nonlocal boundary value problems in 10]). It makes sense to think of the realization of an elliptic operator subject to a coupling condition as a boundary contact problem. Examples are operators of Laplace-type with Kirchhoff or $\delta$-type conditions on a quantum graph (see $[\mathbf{6}, \mathbf{7}$ ), and, in the case of a $\mathbb{Z} / 2$-manifold, operators with transmission or transfer conditions as discussed in the mathematical physics literature, see also [3. One of the motivations for the present work is to contribute to the theoretical underpinning of the heat equation method for these and related problems, specifically as regards the treatment of general elliptic operators of arbitrary order.

Let $E \rightarrow \bar{M}$ be a vector bundle, and let $A \in \operatorname{Diff}^{m}(\bar{M}, E), m>0$, be a differential operator with coefficients in $\operatorname{End}(E)$ (all operators and structures in this work are assumed to be smooth on $\bar{M}$ ). Fix a Riemannian metric on $\bar{M}$ and a Hermitian metric on E. Our main result is the following theorem.

Theorem 1.1. Let $C$ be a coupling condition for $A$, and assume that the boundary contact problem $(A, C)$ is elliptic with parameter in the closed sector $\Lambda \subset \mathbb{C}$ (see Section 1 for details). Then the following holds:

a) The operator $A_{C}=A$ with domain

$$
\mathcal{D}\left(A_{C}\right)=\left\{u \in H^{\operatorname{ord}(A)}(\bar{M}, E) ; C u=0\right\}
$$

is a closed operator in $L^{2}=L^{2}(\bar{M}, E)$.

b) For $\lambda \in \Lambda$ with $|\lambda|>0$ sufficiently large the resolvent $\left(A_{C}-\lambda\right)^{-1}: L^{2} \rightarrow \mathcal{D}\left(A_{C}\right)$ exists and satisfies the norm estimate

$$
\left\|\left(A_{C}-\lambda\right)^{-1}\right\|_{\mathscr{L}\left(L^{2}\right)}=O\left(|\lambda|^{-1}\right)
$$

as $|\lambda| \rightarrow \infty$.

c) For $N>\operatorname{dim} \bar{M} / \operatorname{ord}(A)$ the operator $\left(A_{C}-\lambda\right)^{-N}: L^{2} \rightarrow L^{2}$ is trace class, and for any $\varphi \in C^{\infty}(\bar{M}, \operatorname{End}(E))$ we have an asymptotic expansion

$$
\operatorname{Tr}\left(\varphi\left(A_{C}-\lambda\right)^{-N}\right) \sim|\lambda|^{-N} \sum_{j=0}^{\infty} c_{j}(\hat{\lambda})|\lambda|^{\frac{\operatorname{dim} \bar{M}-j}{\operatorname{ord}(A)}} \quad \text { as }|\lambda| \rightarrow \infty,
$$

where $c_{j}=c_{j}(\varphi, N, A, C) \in C^{\infty}\left(\mathbb{S}^{1} \cap \Lambda\right)$, and $\hat{\lambda}=\lambda /|\lambda|$.

By standard arguments (see $[\mathbf{2}, \mathbf{8}, \mathbf{1 5}$ ) we get the following corollary from Theorem 1.1 .

Corollary 1.3. Let $(A, C)$ be elliptic with parameter in a closed sector of the form $\Lambda=\{\lambda \in \mathbb{C} ;|\arg (\lambda)| \geq \pi / 2-\varepsilon\}$ for some $\varepsilon>0$. Then the following holds:

a) The heat semigroup $e^{-t A_{C}}: L^{2} \rightarrow L^{2}$ exists and is of trace class for $t>0$, and for every $\varphi \in C^{\infty}(\bar{M}, \operatorname{End}(E))$ we have an asymptotic expansion

$$
\operatorname{Tr}\left(\varphi e^{-t A_{C}}\right) \sim \sum_{j=0}^{\infty} \alpha_{j} t^{\frac{j-\operatorname{dim} \bar{M}}{\operatorname{ord}(A)}} \quad \text { as } t \rightarrow 0^{+}
$$

with certain heat invariants $\alpha_{j}=\alpha_{j}(\varphi, A, C)$. 
b) If $A_{C}=A_{C}^{*}>0$, then for every $\varphi \in C^{\infty}(\bar{M}$, $\operatorname{End}(E))$ the zeta function

$$
\zeta\left(s, \varphi, A_{C}\right)=\operatorname{Tr}\left(\varphi A_{C}^{-s}\right)
$$

extends to a meromorphic function on $\mathbb{C}$ with at most simple poles at the points $(\operatorname{dim} \bar{M}-j) / \operatorname{ord}(A), j \in \mathbb{N}_{0}$, and regular on $-\mathbb{N}_{0}$.

c) If $A_{C}=A_{C}^{*}$, then the asymptotics of the eigenvalues $\lambda_{1} \leq \lambda_{2} \leq \ldots$ of $A_{C}$ (counting multiplicities) is given by Weyl's law

$$
\lambda_{k} \sim \text { Const } \cdot k^{\operatorname{ord}(A) / \operatorname{dim} \bar{M}} \text { as } k \rightarrow \infty .
$$

The proof of Theorem 1.1 relies on reducing the nonlocal boundary contact problem $(A, C)$ near $\partial \bar{M}$ to the standard case of a local boundary value problem for a system that is associated with $A$ on $Y \times[0, \varepsilon)$. This utilizes the push-forward map $\wp !$. The expansion then follows by approximating the resolvent of $A_{C}$ by a parametrix in a suitable pseudodifferential calculus that is modelled on Boutet de Monvel's calculus.

\section{Coupling conditions and ellipticity}

Let $U(\partial \bar{M}) \cong \partial \bar{M} \times[0, \varepsilon)$ be a collar neighborhood of the boundary. We extend the covering $\wp: \partial \bar{M} \rightarrow Y$ to a covering $\wp: U(\partial \bar{M}) \rightarrow Y \times[0, \varepsilon)$ in the obvious manner. Choose a Riemannian metric $g_{Y}$ on $Y$, and consider the metric $h=g_{Y}+d x^{2}$ on $Y \times[0, \varepsilon)$. The given metric $g$ on $\bar{M}$ and this choice of metric on $Y \times[0, \varepsilon)$ determine a (discrete) measure $\mu_{(y, x)}$ on the fibre $\wp^{-1}\{(y, x)\}$ for every $y \in Y$ and $0 \leq x<\varepsilon$ so that the canonical map

$$
L_{g}^{2}(U(\partial \bar{M})) \cong L_{h}^{2}(Y \times[0, \varepsilon), \wp ! \mathbb{C})
$$

induced by $\wp$ is unitary. Here $\mathbb{C} \rightarrow U(\partial \bar{M})$ denotes the trivial line bundle, and $\wp ! \mathbb{C} \rightarrow Y \times[0, \varepsilon)$ is the vector bundle with fibre $\wp ! \mathbb{C}_{(y, x)}=L^{2}\left(\wp^{-1}\{(y, x)\}, \mu_{(y, x)}\right)$ for every $y \in Y$ and $x \in[0, \varepsilon)$. Strictly speaking, $\wp ! \mathbb{C}$ is not necessarily a vector bundle since we do not assume that the number of sheets of the covering $\wp: \partial \bar{M} \rightarrow Y$ is the same over each connected component of $Y$, but this is resolved by considering each component separately if necessary. Likewise, consider the push-forward bundle $\wp ! E \rightarrow Y \times[0, \varepsilon)$. For the same reason $\wp$ ! $E$ is not necessarily a bundle over $Y \times[0, \varepsilon)$, but its restriction to $Y_{0} \times[0, \varepsilon)$ is a vector bundle for each connected component $Y_{0}$ of $Y$. The fibre over $(y, x)$ is $\wp ! E_{(y, x)}=L^{2}\left(\wp^{-1}\{(y, x)\}, E\right)$, and the fibrewise $L^{2}$-inner product with respect to the measure $\mu_{(y, x)}$ on $\wp^{-1}\{(y, x)\}$ and the given Hermitian metric on $E$ induces a Hermitian metric on the bundle $\wp ! E$. With this data, the canonical map

$$
\mathscr{U}: L_{g}^{2}(U(\partial \bar{M}), E) \cong L_{h}^{2}(Y \times[0, \varepsilon), \wp ! E)
$$

induced by $\wp$ is unitary. Moreover, $\mathscr{U}$ is an isomorphism

$$
\mathscr{U}: H_{\mathrm{loc}}^{s}(U(\partial \bar{M}), E) \cong H_{\mathrm{loc}}^{s}(Y \times[0, \varepsilon), \wp ! E)
$$

between the Sobolev spaces for all $s \in \mathbb{R}$.

Let $\Lambda \subset \mathbb{C}$ be a closed sector of the form $\Lambda=\left\{r e^{i \varphi} ; r \geq 0,\left|\varphi-\varphi_{0}\right| \leq a\right\}$ for some $a>0$. Let $A \in \operatorname{Diff}^{m}(\bar{M}, E), m>0$. Our standing assumption is that $A$ is elliptic with parameter in $\Lambda$. Recall that this means that the principal symbol

$$
\sigma(A) \in C^{\infty}\left(T^{*} \bar{M} \backslash 0, \operatorname{End}\left(\pi^{*} E\right)\right) \text {, where } \pi: T^{*} \bar{M} \rightarrow \bar{M},
$$

has no eigenvalue in $\Lambda$. 
Definition 2.2. Let $Y_{0}$ be any connected component of $Y$, and let $F_{0, j} \rightarrow Y_{0} \times[0, \varepsilon$, $j=1, \ldots, M_{0}$, be vector bundles. Let $B_{0, j} \in \operatorname{Diff}^{m_{0, j}}\left(Y_{0} \times[0, \varepsilon), \wp ! E, F_{0, j}\right)$ be differential operators, where $m_{0, j}<m$. We call the mapping

$$
C_{0}=\gamma_{Y_{0}}\left(\begin{array}{c}
B_{0,1} \\
\vdots \\
B_{0, M_{0}}
\end{array}\right) \mathscr{U} \circ r_{U_{0}}: C^{\infty}(\bar{M}, E) \rightarrow C^{\infty}\left(Y_{0},\left.\bigoplus_{j=1}^{M_{0}} F_{0, j}\right|_{Y_{0} \times\{0\}}\right)
$$

a coupling condition associated with $Y_{0}$, where $\gamma_{Y_{0}}:\left.f \mapsto f\right|_{Y_{0} \times\{0\}}$ is the trace map for functions on $Y_{0} \times[0, \varepsilon)$, and $r_{U_{0}}: C^{\infty}(\bar{M}, E) \rightarrow C^{\infty}\left(U_{0}, E\right)$ is the restriction of functions to the subset $U_{0}=\wp^{-1}\left(Y_{0} \times[0, \varepsilon)\right)$ of the collar neighborhood of $\partial \bar{M}$. By a coupling condition we mean a map

$$
C: C^{\infty}(\bar{M}, E) \rightarrow \bigoplus_{Y_{0} \subset Y} C^{\infty}\left(Y_{0},\left.\bigoplus_{j=1}^{M_{0}} F_{0, j}\right|_{Y_{0} \times\{0\}}\right)
$$

given by a choice of coupling condition for each component $Y_{0}$.

The mapping

$$
\left(\begin{array}{l}
A \\
C
\end{array}\right): H^{s}(\bar{M}, E) \rightarrow \quad \begin{gathered}
H^{s-m}(\bar{M}, E) \\
\bigoplus \\
\bigoplus_{Y_{0} \subset Y} \bigoplus_{j=1}^{M_{0}} H^{s-m_{0, j}-1 / 2}\left(Y_{0},\left.F_{0, j}\right|_{Y_{0} \times\{0\}}\right)
\end{gathered}
$$

is continuous for all $s>m-1 / 2$. We will refer to the pair $(A, C)$ as a boundary contact problem.

Consider the operator

$$
\mathscr{A}=\mathscr{U} A \mathscr{U}^{-1}: C^{\infty}(Y \times[0, \varepsilon), \wp ! E) \rightarrow C^{\infty}(Y \times[0, \varepsilon), \wp ! E) .
$$

$\mathscr{A} \in \operatorname{Diff}^{m}(Y \times[0, \varepsilon), \wp ! E)$, and $\mathscr{A}$ is elliptic with parameter in $\Lambda$ since this is the case for $A$. Locally, $\mathscr{A}$ can be regarded as a 'diagonal operator' with the various restrictions of $A$ to the sheets of $\wp$ on the diagonal.

Let $\mathscr{B}_{0}=\gamma_{Y_{0}}\left(\begin{array}{lll}B_{0,1} & \cdots & B_{0, M_{0}}\end{array}\right)^{\mathrm{tr}}$. Then

$$
\left(\begin{array}{c}
\mathscr{A} \\
\mathscr{B}_{0}
\end{array}\right): C^{\infty}\left(Y_{0} \times[0, \varepsilon), \wp ! E\right) \rightarrow \begin{gathered}
C^{\infty}\left(Y_{0} \times[0, \varepsilon), \wp ! E\right) \\
C^{\infty}\left(Y_{0},\left.\bigoplus_{j=1}^{M_{0}} F_{0, j}\right|_{Y_{0} \times\{0\}}\right)
\end{gathered}
$$

is a boundary value problem for $\mathscr{A}$ on $Y_{0} \times[0, \varepsilon)$.

Definition 2.4. We call the boundary contact problem $(A, C)$ elliptic with parameter in $\Lambda$ if $A$ is elliptic with parameter in $\Lambda$, and if the boundary value problem (2.3) is elliptic with parameter in $\Lambda$ for all connected components $Y_{0} \subset Y$, i.e., if $\left(\mathscr{A}, \mathscr{B}_{0}\right)$ satisfies the Agmon or parameter-dependent Shapiro-Lopatinsky condition with respect to the sector $\Lambda$. Recall that this means that the boundary symbol

$$
\left(\begin{array}{c}
\sigma(\mathscr{A})\left(y, 0, \eta, D_{x}\right)-\lambda \\
\gamma_{x=0} \sigma\left(\mathscr{B}_{0}\right)\left(y, 0, \eta, D_{x}\right)
\end{array}\right):\left.\mathscr{S}\left(\overline{\mathbb{R}}_{+}\right) \otimes \pi^{*} \wp ! E\right|_{Y_{0} \times\{0\}} \rightarrow \begin{gathered}
\left.\mathscr{S}\left(\overline{\mathbb{R}}_{+}\right) \otimes \pi^{*} \wp ! E\right|_{Y_{0} \times\{0\}} \\
\left.\bigoplus_{j=1}^{M_{0}} \pi^{*} F_{0, j}\right|_{Y_{0} \times\{0\}}
\end{gathered}
$$

is invertible for all $(y, \eta ; \lambda) \in\left(T^{*} Y_{0} \times \Lambda\right) \backslash\{0\}$, where $\pi: T^{*} Y_{0} \rightarrow Y_{0}$ is the canonical projection (see [4, 12] for details). Here $\sigma(\mathscr{A})(y, x, \eta, \xi)$ and $\sigma\left(\mathscr{B}_{0}\right)(y, x, \eta, \xi)$ denote the (vectors) of homogeneous principal symbols of $\mathscr{A}$ and $\mathscr{B}_{0}$, respectively, and $\gamma_{x=0}$ is the evaluation map $f \mapsto f(0)$ on $\mathscr{S}\left(\overline{\mathbb{R}}_{+}\right)$. 
Ellipticity without parameters and the Fredholm property for realizations of elliptic operators subject to coupling conditions has been addressed in [10.

\section{A class of pseudodifferential operators}

In this section we define an enveloping pseudodifferential calculus associated with boundary contact problems. This calculus is modelled on Boutet de Monvel's algebra of pseudodifferential boundary value problems that depend strongly polyhomogeneous on a parameter $\lambda \in \Lambda$ (see [4, 12]). The resolvent of $A_{C}$ will be approximated by a parametrix in this calculus to furnish the proof of Theorem 1.1. see Section 4 .

Let $J_{0, \pm} \rightarrow Y_{0}$ be vector bundles on each connected component $Y_{0} \subset Y$ (the zero bundle is admitted here). Fix a vector field $\partial$ on the double $2 \bar{M}$ of $\bar{M}$ that coincides in the collar neighborhood $\partial \bar{M} \times(-\varepsilon, \varepsilon)$ of the boundary with the vector field $\partial_{x}$, and let

$$
\partial_{+}=r_{+} \nabla_{\partial}^{E} e_{+}: C^{\infty}(\bar{M}, E) \rightarrow C^{\infty}(\bar{M}, E),
$$

where $e_{+}$is the trivial extension operator by zero for functions defined on $M$ to the double $2 \bar{M}, r_{+}$is the restriction operator for distributions on $2 \bar{M}$ to $M$, and $\nabla^{E}$ is a Hermitian connection on the (extended) bundle $E \rightarrow 2 \bar{M}$.

Moreover, let $\ell \in \mathbb{N}$ be fixed. $\ell$ represents the anisotropy between the covariables and the parameter $\lambda \in \Lambda$. For the treatment of the resolvent of $A_{C}$ we will choose $\ell=m=\operatorname{ord}(A)$.

Definition 3.1 (Regularizing Green operators). a) By $\Psi^{-\infty, 0}(\Lambda)$ we denote the class of all operator families

$$
G(\lambda): \begin{gathered}
H_{0}^{s}(\bar{M}, E) \\
\oplus \\
\bigoplus_{Y_{0} \subset Y} H^{s}\left(Y_{0}, J_{0,-}\right)
\end{gathered} \rightarrow \underset{Y_{0} \subset Y}{\bigoplus^{t}(\bar{M}, E)} \begin{gathered}
\oplus \\
H^{t}\left(Y_{0}, J_{0,+}\right)
\end{gathered}
$$

that depend rapidly decreasing on $\lambda \in \Lambda$, for all $s, t \in \mathbb{R}$. In other words, $\Psi^{\infty, 0}(\Lambda)$ consists of all operator families with $C^{\infty}$-kernels that depend rapidly decreasing on $\lambda \in \Lambda$.

b) For $d \in \mathbb{N}_{0}$ let $\Psi^{-\infty, d}(\Lambda)$ be the class of all operator families of the form

$$
G(\lambda)=\sum_{j=0}^{d} G_{j}(\lambda)\left(\begin{array}{cc}
\partial_{+} & 0 \\
0 & 0
\end{array}\right)^{j}: \begin{gathered}
C^{\infty}(\bar{M}, E) \\
\oplus \\
\underset{Y_{0} \subset Y}{\oplus} C^{\infty}\left(Y_{0}, J_{0,-}\right)
\end{gathered} \rightarrow \underset{Y_{0} \subset Y}{\bigoplus_{c}^{\infty} C^{\infty}\left(Y_{0}, J_{0,+}\right)}
$$

with $G_{j}(\lambda) \in \Psi^{-\infty, 0}(\Lambda)$.

Let $\varphi \in C^{\infty}(Y \times[0, \varepsilon))$ be such that $\varphi$ is locally constant on $Y=Y \times\{0\}$. The restriction of $\varphi$ to $Y$ is a sum

$$
\left.\varphi\right|_{Y}=\sum_{Y_{0} \subset Y} a_{Y_{0}} \cdot \chi_{Y_{0}}
$$

of multiples of the characteristic functions $\chi_{Y_{0}}$ associated with the various connected components $Y_{0}$ of $Y$. Since multiplication by the characteristic function $\chi_{Y_{1}}$ is a projection operator, it thus makes sense to consider

$$
\chi_{Y_{1}}: \bigoplus_{Y_{0} \subset Y} C^{\infty}\left(Y_{0}, J_{0, \pm}\right) \rightarrow C^{\infty}\left(Y_{1}, J_{1, \pm}\right)
$$


as the projection operator to the subspace

$$
C^{\infty}\left(Y_{1}, J_{1, \pm}\right) \hookrightarrow \bigoplus_{Y_{0} \subset Y} C^{\infty}\left(Y_{0}, J_{0, \pm}\right)
$$

Consequently, we consider

$$
\left.\varphi\right|_{Y}: \bigoplus_{Y_{0} \subset Y} C^{\infty}\left(Y_{0}, J_{0, \pm}\right) \rightarrow \bigoplus_{Y_{0} \subset Y} C^{\infty}\left(Y_{0}, J_{0, \pm}\right)
$$

an operator defined by the sum of multiples (3.2) of the projection operators associated with the characteristic functions of the connected components.

Let $\tilde{\varphi} \in C^{\infty}(\bar{M})$ be such that $\tilde{\varphi}$ is the pull-back $\wp^{*} \varphi$ for a function $\varphi$ as above near the boundary $\partial \bar{M}$. For such functions $\tilde{\varphi}$ we are going to use the notational convention that $\tilde{\varphi}$ is also to be understood as the operator

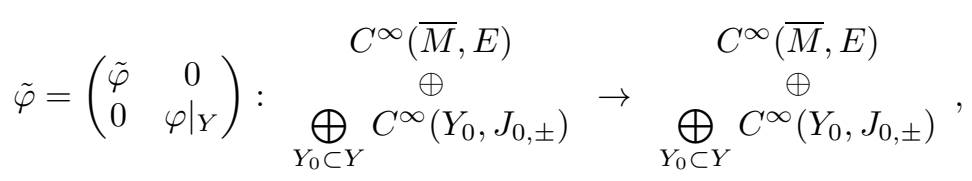

given by the multiplication operator with the function $\tilde{\varphi}$ in the upper left corner, and in the lower right corner by the operator $\left.\varphi\right|_{Y}$ explained above.

Definition 3.3 (Singular Green operators). Let $\mu \in \mathbb{Z}, d \in \mathbb{N}_{0}$. The class $\Psi_{G}^{\mu, d}(\Lambda)$ consists of all operator families

$$
G(\lambda): \begin{gathered}
C^{\infty}(\bar{M}, E) \\
\oplus \\
\bigoplus_{Y_{0} \subset Y} C^{\infty}\left(Y_{0}, J_{0,-}\right)
\end{gathered} \rightarrow \underset{Y_{0} \subset Y}{\bigoplus^{\infty}(\bar{M}, E)} \stackrel{\oplus}{\oplus} C^{\infty}\left(Y_{0}, J_{0,+}\right)
$$

with the following properties:

- Let $Y_{0} \subset Y$ be any connected component, and let $\varphi, \psi \in C^{\infty}\left(Y_{0} \times[0, \varepsilon)\right)$ be compactly supported and constant on $Y_{0}$. Consider the operator family

$$
\left(\begin{array}{cc}
\mathscr{U} & 0 \\
0 & 1
\end{array}\right) \circ\left(\wp^{*} \varphi G(\lambda) \wp^{*} \psi\right) \circ\left(\begin{array}{cc}
\mathscr{U}^{-1} & 0 \\
0 & 1
\end{array}\right),
$$

acting in the spaces

$$
\begin{array}{ccc}
C^{\infty}\left(Y_{0} \times[0, \varepsilon), \wp ! E\right) & & C^{\infty}\left(Y_{0} \times[0, \varepsilon), \wp ! E\right) \\
C^{\infty}\left(Y_{0}, J_{0,-}\right) & \rightarrow & \oplus \\
& & C^{\infty}\left(Y_{0}, J_{0,+}\right)
\end{array}
$$

We require this family to belong to the class of (strongly polyhomogeneous) anisotropic parameter-dependent (generalized) singular Green operators of order $\mu$ and type $d$ in Boutet de Monvel's calculus on $Y_{0} \times[0, \varepsilon)$.

- Let $\tilde{\varphi}, \tilde{\psi} \in C^{\infty}(\bar{M})$ be such that $\tilde{\varphi}=\wp^{*} \varphi$ and $\tilde{\psi}=\wp^{*} \psi$ near the boundary $\partial \bar{M}$ for functions $\varphi, \psi \in C^{\infty}(Y \times[0, \varepsilon))$ that are locally constant on $Y$, and assume that $\operatorname{supp} \varphi \cap \operatorname{supp} \psi \cap Y=\emptyset$.

We then require the operator family $\tilde{\varphi} G(\lambda) \tilde{\psi}$ to belong to the class $\Psi^{-\infty, d}(\Lambda)$ defined in Definition 3.1 
Since the operator family (3.4) belongs to Boutet de Monvel's calculus on $Y_{0} \times[0, \varepsilon$ ) it has a principal boundary symbol associated with it. Let $\sigma_{Y_{0}}(G)(y, \eta ; \lambda)$ be that principal boundary symbol, an element of

$$
C^{\infty}\left(\left(T^{*} Y_{0} \times \Lambda\right) \backslash\{0\}, \operatorname{Hom}\left(\begin{array}{cc}
\left.\mathscr{S}\left(\overline{\mathbb{R}}_{+}\right) \otimes \pi^{*} \wp ! E\right|_{Y_{0}} & \left.\mathscr{S}\left(\overline{\mathbb{R}}_{+}\right) \otimes \pi^{*} \wp ! E\right|_{Y_{0}} \\
\oplus & \oplus \\
\pi^{*} J_{0,-} & \pi^{*} J_{0,+}
\end{array}\right)\right),
$$

where $\pi:\left(T^{*} Y_{0} \times \Lambda\right) \backslash\{0\} \rightarrow Y_{0}$ is the canonical projection. Thus, associated with $G(\lambda)$, we have an operator valued principal $Y_{0}$-symbol on $\left(T^{*} Y_{0} \times \Lambda\right) \backslash\{0\}$ for all connected components $Y_{0} \subset Y$. Recall that $\sigma_{Y_{0}}(G)(y, \eta ; \lambda)$ is homogeneous in the sense that $\sigma_{Y_{0}}(G)\left(y, \varrho \eta ; \varrho^{\ell} \lambda\right)$ equals

$$
\varrho^{\mu}\left(\begin{array}{cc}
\left.\kappa_{\varrho} \otimes \operatorname{Id}_{\pi^{*} \wp ! E}\right|_{Y_{0}} & 0 \\
0 & \operatorname{Id}_{\pi^{*} J_{0,+}}
\end{array}\right) \sigma_{Y_{0}}(G)(y, \eta ; \lambda)\left(\begin{array}{cc}
\left.\kappa_{\varrho}^{-1} \otimes \operatorname{Id}_{\pi^{*} \wp ! E}\right|_{Y_{0}} & 0 \\
0 & \operatorname{Id}_{\pi^{*} J_{0,-}}
\end{array}\right)
$$

for $\varrho>0$, where $\left(\kappa_{\varrho} u\right)(x)=\varrho^{1 / 2} u(\varrho x)$.

Remark 3.5. In local coordinates near the boundary of $Y_{0} \times[0, \varepsilon)$, the local boundary symbols of the operator (3.4) are operator families

$$
g(y, \eta ; \lambda)=\sum_{j=0}^{d} g_{j}(y, \eta ; \lambda)\left(\begin{array}{cc}
\partial_{+} & 0 \\
0 & 0
\end{array}\right)^{j}
$$

where the $g_{j}(y, \eta ; \lambda)$ are boundary symbols of order $\mu-j$ and type zero.

A boundary symbol of order $\mu \in \mathbb{Z}$ and type zero is a $C^{\infty}$-function

$$
\begin{aligned}
& \mathscr{S}^{\prime}\left(\overline{\mathbb{R}}_{+}\right) \otimes \mathbb{C}^{\left.\operatorname{dim} \wp ! E\right|_{Y_{0}}} \quad \mathscr{S}\left(\overline{\mathbb{R}}_{+}\right) \otimes \mathbb{C}^{\left.\operatorname{dim} \wp ! E\right|_{Y_{0}}} \\
& h(y, \eta ; \lambda): \quad \stackrel{\oplus}{\mathbb{C}^{\operatorname{dim} J_{0,-}}} \quad \rightarrow \quad \stackrel{\oplus}{\mathbb{C}^{\operatorname{dim} J_{0,+}}}
\end{aligned}
$$

such that

$$
\left(\begin{array}{cc}
\kappa_{\left(1+|\eta|+|\lambda|^{1 / \ell}\right)}^{-1} & 0 \\
0 & 1
\end{array}\right)\left(\partial_{y}^{\alpha} \partial_{\eta}^{\beta} \partial_{\lambda}^{\gamma} h(y, \eta ; \lambda)\right)\left(\begin{array}{cc}
\kappa_{\left(1+|\eta|+|\lambda|^{1 / \ell}\right)} & 0 \\
0 & 1
\end{array}\right)
$$

is $O\left(\left(1+|\eta|+|\lambda|^{1 / \ell}\right)^{\mu-|\beta|-\ell|\gamma|}\right)$ as $|(\eta, \lambda)| \rightarrow \infty$ in the topology of uniform convergence on bounded subsets of the continuous operators in the spaces (3.6), uniformly for $y$ in compact subsets. Moreover, $h(y, \eta ; \lambda)$ has an asymptotic expansion

$$
h(y, \eta ; \lambda) \sim \sum_{j=0}^{\infty} \chi(\eta, \lambda) h_{(\mu-j)}(y, \eta ; \lambda),
$$

where $\chi$ is an excision function of the origin, and the operator family $h_{(\mu-j)}(y, \eta ; \lambda)$ is (twisted) anisotropic homogeneous of degree $\mu-j$ in the sense that

$$
h_{(\mu-j)}\left(y, \varrho \eta ; \varrho^{\ell} \lambda\right)=\varrho^{\mu-j}\left(\begin{array}{cc}
\kappa_{\varrho} & 0 \\
0 & 1
\end{array}\right) h_{(\mu-j)}(y, \eta ; \lambda)\left(\begin{array}{cc}
\kappa_{\varrho}^{-1} & 0 \\
0 & 1
\end{array}\right)
$$

for $\varrho>0$ and $(\eta, \lambda) \neq(0,0)$.

This description of the boundary symbol structure of generalized singular Green operators in Boutet de Monvel's calculus follows Schulze [12, see also [11.

Let

$$
h_{0}(y, \eta ; \lambda): \mathscr{S}^{\prime}\left(\overline{\mathbb{R}}_{+}\right) \otimes \mathbb{C}^{\left.\operatorname{dim} \wp ! E\right|_{Y_{0}}} \rightarrow \mathscr{S}\left(\overline{\mathbb{R}}_{+}\right) \otimes \mathbb{C}^{\left.\operatorname{dim} \wp ! E\right|_{Y_{0}}}
$$

be the upper left corner of the symbol (3.6). From the latter description it is immediately clear that $h_{0}(y, \eta ; \lambda)$ is of trace class as an operator acting in $L^{2}\left(\overline{\mathbb{R}}_{+}\right) \otimes$ 
$\mathbb{C}^{\left.\operatorname{dim} \wp ! E\right|_{Y_{0}}}$, and that its trace $\operatorname{tr}_{L^{2}} h_{0}(y, \eta ; \lambda)$ is an ordinary anisotropic parameterdependent classical symbol of order $\mu$ (see Remark 3.10).

Remark 3.7. Let $\mathscr{B}_{G}^{\mu, d}(\Lambda)$ be the class of anisotropic parameter-dependent singular Green operators $G(\lambda): C^{\infty}(\bar{M}, E) \rightarrow C^{\infty}(\bar{M}, E)$ of order $\mu$ and type $d$ in Boutet de Monvel's calculus on $\bar{M}$, and let $\mathscr{B}^{-\infty, d}(\Lambda)$ be the subspace of regularizing singular Green operators of type $d$. Then

$$
\mathscr{B}_{G}^{\mu, d}(\Lambda) \subset \Psi_{G}^{\mu, d}(\Lambda) \text { and } \mathscr{B}^{-\infty, d}(\Lambda)=\Psi^{-\infty, d}(\Lambda) .
$$

In the interesting cases for us $\mathscr{B}_{G}^{\mu, d}(\Lambda) \neq \Psi_{G}^{\mu, d}(\Lambda)$ because the covering $\wp: \partial \bar{M} \rightarrow Y$ has multiple sheets.

Definition 3.8 (The full calculus). Let $\mu \in \mathbb{Z}, d \in \mathbb{N}_{0}$. The class $\Psi^{\mu, d}(\Lambda)$ consists of all operator families

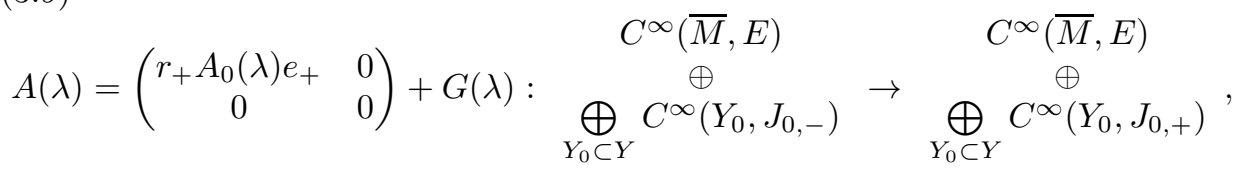

where $G(\lambda) \in \Psi_{G}^{\mu, d}(\Lambda)$, and $A_{0}(\lambda)$ is an anisotropic parameter-dependent pseudodifferential operator on $2 \bar{M}$ with the transmission property at $\partial \bar{M}$.

Remark 3.10. The local symbols $a(z, \zeta ; \lambda)$ of $A_{0}(\lambda)$ in Definition 3.8 satisfy the symbol estimates

$$
\left|\partial_{z}^{\alpha} \partial_{\zeta}^{\beta} \partial_{\lambda}^{\gamma} a(z, \zeta ; \lambda)\right|=O\left(\left(1+|\zeta|+|\lambda|^{1 / \ell}\right)^{\mu-|\beta|-\ell|\gamma|}\right)
$$

as $|(\zeta, \lambda)| \rightarrow \infty$, uniformly for $z$ in compact subsets, and they have an asymptotic expansion

$$
a(z, \zeta ; \lambda) \sim \sum_{j=0}^{\infty} \chi(\zeta, \lambda) a_{(\mu-j)}(z, \zeta ; \lambda),
$$

where $\chi$ is an excision function of the origin, and $a_{(\mu-j)}(z, \zeta ; \lambda)$ is anisotropic homogeneous of degree $\mu-j$, i.e.,

$$
a_{(\mu-j)}\left(z, \varrho \zeta ; \varrho^{\ell} \lambda\right)=\varrho^{\mu-j} a_{(\mu-j)}(z, \zeta ; \lambda) \text { for } \varrho>0 \text { and }(\zeta, \lambda) \neq(0,0) .
$$

Let $\varphi \in C^{\infty}\left(Y_{0} \times[0, \varepsilon)\right)$ be compactly supported such that $\varphi \equiv 1$ near $Y_{0}$.

$$
\mathscr{U} \circ\left(\wp^{*} \varphi r_{+} A_{0}(\lambda) e_{+} \wp^{*} \varphi\right) \circ \mathscr{U}^{-1}: C^{\infty}\left(Y_{0} \times[0, \varepsilon), \wp ! E\right) \rightarrow C^{\infty}\left(Y_{0} \times[0, \varepsilon), \wp ! E\right)
$$

is a parameter-dependent pseudodifferential operator in Boutet de Monvel's calculus on $Y_{0} \times[0, \varepsilon)$, and thus has a principal boundary symbol. For each connected component $Y_{0} \subset Y$, let $\sigma_{Y_{0}}\left(A_{0}\right)(y, \eta ; \lambda)$ be that principal boundary symbol on $\left(T^{*} Y_{0} \times \Lambda\right) \backslash\{0\}$. Consequently, the following principal symbols are associated with every operator $A(\lambda) \in \Psi^{\mu, d}(\Lambda)$ as given by (3.9):

- The homogeneous principal symbol

$\sigma(A)(z, \zeta ; \lambda):=\sigma\left(A_{0}\right)(z, \zeta ; \lambda) \in C^{\infty}\left(\left(T^{*} \bar{M} \times \Lambda\right) \backslash\{0\}, \operatorname{End}\left(\pi^{*} E\right)\right)$,

where $\pi:\left(T^{*} \bar{M} \times \Lambda\right) \backslash\{0\} \rightarrow \bar{M}$ is the canonical projection. 
- The principal $Y_{0}$-symbol

$$
\sigma_{Y_{0}}(A)(y, \eta ; \lambda):=\left(\begin{array}{cc}
\sigma_{Y_{0}}\left(A_{0}\right)(y, \eta ; \lambda) & 0 \\
0 & 0
\end{array}\right)+\sigma_{Y_{0}}(G)(y, \eta ; \lambda)
$$

defined on $\left(T^{*} Y_{0} \times \Lambda\right) \backslash\{0\}$ for every connected component $Y_{0} \subset Y$.

Definition 3.11. An operator family $A(\lambda) \in \Psi^{\mu, d}(\Lambda)$ is parameter-dependent elliptic if its homogeneous principal symbol $\sigma(A)(z, \zeta ; \lambda)$ is invertible for every $(z, \zeta ; \lambda) \in\left(T^{*} \bar{M} \times \Lambda\right) \backslash\{0\}$, and its principal $Y_{0}$-symbol $\sigma_{Y_{0}}(A)(y, \eta ; \lambda)$ is invertible for all $(y, \eta ; \lambda) \in\left(T^{*} Y_{0} \times \Lambda\right) \backslash\{0\}$, for all connected components $Y_{0} \subset Y$.

Theorem 3.12. a) Every $A(\lambda) \in \Psi^{\mu, d}(\Lambda)$ extends by continuity to a family of continuous operators

$$
A(\lambda): \begin{gathered}
H^{s}(\bar{M}, E) \\
\oplus \\
\bigoplus_{Y_{0} \subset Y} H^{s}\left(Y_{0}, J_{0,-}\right)
\end{gathered} \rightarrow \underset{Y_{0} \subset Y}{\bigoplus^{s-\mu}(\bar{M}, E)} H^{s-\mu}\left(Y_{0}, J_{0,+}\right)
$$

for $s>d-1 / 2$.

b) Let $A(\lambda) \in \Psi^{\mu, 0}(\Lambda)$, where $\mu \leq 0$. Then the operator norm of

$$
A(\lambda): \begin{gathered}
L^{2}(\bar{M}, E) \\
\oplus \\
\bigoplus_{Y_{0} \subset Y} L^{2}\left(Y_{0}, J_{0,-}\right)
\end{gathered} \rightarrow \underset{Y_{0} \subset Y}{\bigoplus^{2}(\bar{M}, E)} \stackrel{\oplus}{\oplus} L^{2}\left(Y_{0}, J_{0,+}\right)
$$

is $O\left(|\lambda|^{\mu / \ell}\right)$ as $|\lambda| \rightarrow \infty$.

Proof. Write

$$
A(\lambda)=\left(\begin{array}{cc}
r_{+} A_{0}(\lambda) e_{+} & 0 \\
0 & 0
\end{array}\right)+G(\lambda)
$$

as in (3.9). Both a) and b) are clear for $\left(\begin{array}{cc}r_{+} A_{0}(\lambda) e_{+} & 0 \\ 0 & 0\end{array}\right)$.

Let $\varphi_{0}, \psi_{0} \in C^{\infty}\left(Y_{0} \times[0, \varepsilon)\right)$ be compactly supported such that $\varphi_{0} \equiv 1$ near $Y_{0}$, and $\psi_{0} \equiv 1$ in a neighborhood of the support of $\varphi_{0}$. Then

$$
G(\lambda)=\sum_{Y_{0} \subset Y} \wp^{*} \varphi_{0} G(\lambda) \wp^{*} \psi_{0}+R(\lambda),
$$

where $R(\lambda) \in \Psi^{-\infty, d}(\Lambda)$. Both a) and b) are evident for $R(\lambda)$. The operator

$$
\left(\begin{array}{cc}
\mathscr{U} & 0 \\
0 & 1
\end{array}\right) \circ\left(\wp^{*} \varphi_{0} G(\lambda) \wp^{*} \psi_{0}\right) \circ\left(\begin{array}{cc}
\mathscr{U}^{-1} & 0 \\
0 & 1
\end{array}\right)
$$

is a parameter-dependent generalized singular Green operator in Boutet de Monvel's calculus on $Y_{0} \times[0, \varepsilon)$ (supported near the boundary $Y_{0}$ ). Consequently, both assertions a) and b) are valid for this operator on $Y_{0} \times[0, \varepsilon)$. Since the canonical map $\mathscr{U}$ is an isometry in $L^{2}$ and an isomorphism between the Sobolev spaces, see the discussion around (2.1), a) and b) follow for the operators $\wp^{*} \varphi_{0} G(\lambda) \wp^{*} \psi_{0}$.

Theorem 3.14. Let $A_{j}(\lambda) \in \Psi^{\mu_{j}, d_{j}}(\Lambda), j=1,2$, and assume that the vector bundles fit together such that the composition $A_{1}(\lambda) A_{2}(\lambda)$ is defined.

Then $A_{1}(\lambda) A_{2}(\lambda) \in \Psi^{\mu_{1}+\mu_{2}, d}(\Lambda)$, where $d=\max \left\{d_{1}+\mu_{2}, d_{2}\right\}$. We have

$$
\begin{aligned}
\sigma\left(A_{1} A_{2}\right)(z, \zeta ; \lambda) & =\sigma\left(A_{1}\right)(z, \zeta ; \lambda) \sigma\left(A_{2}\right)(z, \zeta ; \lambda), \\
\sigma_{Y_{0}}\left(A_{1} A_{2}\right)(y, \eta ; \lambda) & =\sigma_{Y_{0}}\left(A_{1}\right)(y, \eta ; \lambda) \sigma_{Y_{0}}\left(A_{2}\right)(y, \eta ; \lambda)
\end{aligned}
$$


for all connected components $Y_{0} \subset Y$.

Proof. We first observe that the composition of operator families is welldefined in

$$
\begin{aligned}
& \Psi^{\mu_{1}, d_{1}}(\Lambda) \times \Psi^{-\infty, d_{2}}(\Lambda) \rightarrow \Psi^{-\infty, d}(\Lambda), \\
& \Psi^{-\infty, d_{1}}(\Lambda) \times \Psi^{\mu_{2}, d_{2}}(\Lambda) \rightarrow \Psi^{-\infty, d}(\Lambda) .
\end{aligned}
$$

The first of these statements follows immediately from the definition of the calculus and Theorem 3.12. To prove the second, let $\varphi_{0}, \psi_{0} \in C^{\infty}\left(Y_{0} \times[0, \varepsilon)\right)$ be compactly supported, and let $\varphi_{0} \equiv 1$ near $Y_{0}$, and $\psi_{0} \equiv 1$ in a neighborhood of the support of $\varphi_{0}$. Let

$$
\phi_{\text {int }}=1-\sum_{Y_{0} \subset Y} \wp^{*} \varphi_{0}
$$

and let $\psi_{\text {int }} \in C^{\infty}(\bar{M})$ be compactly supported away from $\partial \bar{M}$ with $\psi_{\text {int }} \equiv 1$ in a neighborhood of the support of $\varphi_{\text {int }}$. Let $A(\lambda) \in \Psi^{\mu_{2}, d_{2}}(\Lambda)$. We write

$$
A(\lambda)=\sum_{Y_{0} \subset Y} \wp^{*} \varphi_{0} A(\lambda) \wp^{*} \psi_{0}+\varphi_{\mathrm{int}} A(\lambda) \psi_{\mathrm{int}}+R(\lambda)
$$

with $R(\lambda) \in \Psi^{-\infty, d_{2}}(\Lambda)$. Thus it remains to treat the composition of $G(\lambda) \in$ $\Psi^{-\infty, d_{1}}(\Lambda)$ with every summand in (3.16). Write

$$
G(\lambda)=\sum_{j=0}^{d_{1}} G_{j}(\lambda)\left(\begin{array}{cc}
\partial_{+} & 0 \\
0 & 0
\end{array}\right)^{j} \text { with } G_{j}(\lambda) \in \Psi^{-\infty, 0}(\Lambda) .
$$

From the composition theorem in Boutet de Monvel's calculus on $Y_{0} \times[0, \varepsilon)$ we obtain that

$$
\left(\begin{array}{cc}
\mathscr{U} & 0 \\
0 & 1
\end{array}\right)\left(\begin{array}{cc}
\partial_{+} & 0 \\
0 & 0
\end{array}\right)^{j}\left(\begin{array}{cc}
\mathscr{U}^{-1} & 0 \\
0 & 1
\end{array}\right) \circ\left(\begin{array}{cc}
\mathscr{U} & 0 \\
0 & 1
\end{array}\right) \wp^{*} \varphi_{0} A(\lambda) \wp^{*} \psi_{0}\left(\begin{array}{cc}
\mathscr{U}^{-1} & 0 \\
0 & 1
\end{array}\right)
$$

belongs to Boutet de Monvel's calculus on $Y_{0} \times[0, \varepsilon)$. Since $G_{j}(\lambda)$ is an integral operator with $C^{\infty}$-kernel that depends rapidly decreasing on $\lambda \in \Lambda$, we thus conclude that

$$
G_{j}(\lambda)\left(\begin{array}{cc}
\partial_{+} & 0 \\
0 & 0
\end{array}\right)^{j} \wp^{*} \varphi_{0} A(\lambda) \wp^{*} \psi_{0} \in \Psi^{-\infty, d}(\Lambda) .
$$

The latter conclusion utilizes the mapping properties of $\mathscr{U}$, and the standard mapping properties of operators in Boutet de Monvel's calculus. It is clear that the compositions $G(\lambda) \varphi_{\mathrm{int}} A(\lambda) \psi_{\mathrm{int}}$ and $G(\lambda) R(\lambda)$ belong to $\Psi^{-\infty, d}(\Lambda)$. This finishes the proof of (3.15).

Now consider the general case. Let $\tilde{\psi}_{0} \in C^{\infty}\left(Y_{0} \times[0, \varepsilon)\right)$ be compactly supported with $\tilde{\psi}_{0} \equiv 1$ in a neighborhood of the support of $\psi_{0}$. Write

$$
\begin{aligned}
\wp^{*} \varphi_{0} A_{1}(\lambda) A_{2}(\lambda) \wp^{*} \psi_{0}=\left(\wp^{*} \varphi_{0} A_{1}(\lambda) \wp^{*} \psi_{0}\right)\left(\wp^{*} \tilde{\psi}_{0} A_{2}(\lambda) \wp^{*} \psi_{0}\right) \\
+\wp^{*} \varphi_{0} A_{1}(\lambda)\left(1-\wp^{*} \tilde{\psi}_{0}\right) A_{2}(\lambda) \wp^{*} \psi_{0} .
\end{aligned}
$$

The operator $\left(1-\wp^{*} \tilde{\psi}_{0}\right) A_{2}(\lambda) \wp^{*} \psi_{0} \in \Psi^{-\infty, d_{2}}(\Lambda)$, and thus

$$
\wp^{*} \varphi_{0} A_{1}(\lambda)\left(1-\wp^{*} \tilde{\psi}_{0}\right) A_{2}(\lambda) \wp^{*} \psi_{0} \in \Psi^{-\infty, d}(\Lambda)
$$

by (3.15). Utilizing the mapping $\mathscr{U}$ and the composition theorem in Boutet de Monvel's calculus on $Y_{0} \times[0, \varepsilon)$, we get

$$
\left(\wp^{*} \varphi_{0} A_{1}(\lambda) \wp^{*} \psi_{0}\right)\left(\wp^{*} \tilde{\psi}_{0} A_{2}(\lambda) \wp^{*} \psi_{0}\right) \in \Psi^{\mu_{1}+\mu_{2}, d}(\Lambda) .
$$


Hence $\wp^{*} \varphi_{0} A_{1}(\lambda) A_{2}(\lambda) \wp^{*} \psi_{0} \in \Psi^{\mu_{1}+\mu_{2}, d}(\Lambda)$, and

$$
\begin{aligned}
\sigma\left(\wp^{*} \varphi_{0} A_{1}(\lambda) A_{2}(\lambda) \wp^{*} \psi_{0}\right) & =\wp^{*} \varphi_{0} \sigma\left(A_{1}\right) \sigma\left(A_{2}\right), \\
\sigma_{Y_{0}}\left(\wp^{*} \varphi_{0} A_{1}(\lambda) A_{2}(\lambda) \wp^{*} \psi_{0}\right) & =\sigma_{Y_{0}}\left(A_{1} A_{2}\right)=\sigma_{Y_{0}}\left(A_{1}\right) \sigma_{Y_{0}}\left(A_{2}\right) .
\end{aligned}
$$

One comment about this argument is in order. Decompose the $A_{j}(\lambda)$ according to (3.9) into pseudodifferential and singular Green parts. The pseudodifferential parts multiply by the composition theorem in Boutet de Monvel's calculus on $\bar{M}$. Hence we have the pseudodifferential parts of $\wp^{*} \varphi_{0} A_{1}(\lambda) A_{2}(\lambda) \wp^{*} \psi_{0}$ under control, we don't catch contributions that are not pseudolocal on $\bar{M}$ by pulling back pseudodifferential operators from $Y_{0} \times[0, \varepsilon)$ via $\mathscr{U}$.

Let $\hat{\psi}_{0} \in C^{\infty}\left(Y_{0} \times[0, \varepsilon)\right)$ be compactly supported such that $\hat{\psi}_{0} \equiv 1$ in a neighborhood of the support of $\varphi_{0}$, and such that $\psi_{0} \equiv 1$ in a neighborhood of the support of $\hat{\psi}_{0}$. Then

$$
\begin{aligned}
\wp^{*} \varphi_{0} A_{1}(\lambda) A_{2}(\lambda)\left(1-\wp^{*} \psi_{0}\right)=\left(\wp^{*} \varphi_{0} A_{1}(\lambda)\left(1-\wp^{*} \hat{\psi}_{0}\right)\right) A_{2}(\lambda)\left(1-\wp^{*} \psi_{0}\right) \\
+\wp^{*} \varphi_{0} A_{1}(\lambda)\left(\wp^{*} \hat{\psi}_{0} A_{2}(\lambda)\left(1-\wp^{*} \psi_{0}\right)\right) .
\end{aligned}
$$

Now

$$
\left(\wp^{*} \varphi_{0} A_{1}(\lambda)\left(1-\wp^{*} \hat{\psi}_{0}\right)\right) \in \Psi^{-\infty, d_{1}}(\Lambda) \text { and }\left(\wp^{*} \hat{\psi}_{0} A_{2}(\lambda)\left(1-\wp^{*} \psi_{0}\right)\right) \in \Psi^{-\infty, d_{2}}(\Lambda),
$$

and thus

$$
\wp^{*} \varphi_{0} A_{1}(\lambda) A_{2}(\lambda)\left(1-\wp^{*} \psi_{0}\right) \in \Psi^{-\infty, d}(\Lambda)
$$

by (3.15). A similar argument shows that

$$
\varphi_{\text {int }} A_{1}(\lambda) A_{2}(\lambda) \psi_{\text {int }} \in \Psi^{\mu_{1}+\mu_{2}, d}(\Lambda)
$$

with

$$
\sigma\left(\varphi_{\mathrm{int}} A_{1}(\lambda) A_{2}(\lambda) \psi_{\mathrm{int}}\right)=\varphi_{\mathrm{int}} \sigma\left(A_{1}\right) \sigma\left(A_{2}\right)
$$

and likewise

$$
\varphi_{\mathrm{int}} A_{1}(\lambda) A_{2}(\lambda)\left(1-\psi_{\mathrm{int}}\right) \in \Psi^{-\infty, d}(\Lambda)
$$

Now write

$$
A_{1}(\lambda) A_{2}(\lambda)=\sum_{Y_{0} \subset Y} \wp^{*} \varphi_{0} A_{1}(\lambda) A_{2}(\lambda) \wp^{*} \psi_{0}+\varphi_{\text {int }} A_{1}(\lambda) A_{2}(\lambda) \psi_{\text {int }}+R(\lambda) .
$$

According to the arguments given above we conclude that $R(\lambda) \in \Psi^{-\infty, d}(\Lambda)$, and we see that every summand in this representation belongs to $\Psi^{\mu_{1}+\mu_{2}, d}(\Lambda)$. Consequently, $A_{1}(\lambda) A_{2}(\lambda) \in \Psi^{\mu_{1}+\mu_{2}, d}(\Lambda)$, and the asserted identities for the principal symbols follow from the corresponding identities obtained above for the summands. This finishes the proof of the theorem.

Theorem 3.17. Let $A(\lambda) \in \Psi^{\mu, d}(\Lambda)$ be parameter-dependent elliptic in the sense of Definition 3.11. Then there exists a parameter-dependent parametrix $P(\lambda) \in$ $\Psi^{-\mu,(d-\mu)_{+}}(\Lambda)$ of $A(\lambda)$, where $(d-\mu)_{+}=\max \{d-\mu, 0\}$, i.e., we have

$$
P(\lambda) A(\lambda)-1 \in \Psi^{-\infty, *}(\Lambda) \text {, and } A(\lambda) P(\lambda)-1 \in \Psi^{-\infty, *}(\Lambda) .
$$

The types of these regularizing remainders are given by the type formula from Theorem 3.14 . 
Proof. Consider the restrictions of $A(\lambda)$ to $\wp^{-1}\left(Y_{0} \times[0, \varepsilon)\right)$, i.e., the operators

$$
\left.A(\lambda)\right|_{\wp^{-1}\left(Y_{0} \times[0, \varepsilon)\right)}: \begin{gathered}
C_{c}^{\infty}\left(\wp^{-1}\left(Y_{0} \times[0, \varepsilon)\right), E\right) \\
\oplus \\
C^{\infty}\left(Y_{0}, J_{0,-}\right)
\end{gathered} \rightarrow \begin{gathered}
C^{\infty}\left(\wp^{-1}\left(Y_{0} \times[0, \varepsilon)\right), E\right) \\
\oplus \\
C^{\infty}\left(Y_{0}, J_{0,+}\right)
\end{gathered}
$$

By construction of the calculus and by assumption, we obtain that

$$
\left.\left(\begin{array}{cc}
\mathscr{U} & 0 \\
0 & 1
\end{array}\right) A(\lambda)\right|_{\wp^{-1}\left(Y_{0} \times[0, \varepsilon)\right)}\left(\begin{array}{cc}
\mathscr{U}^{-1} & 0 \\
0 & 1
\end{array}\right)
$$

is a parameter-dependent elliptic boundary value problem in Boutet de Monvel's calculus on $Y_{0} \times[0, \varepsilon)$. Let $\mathscr{P}_{Y_{0}}(\lambda)$ be a parameter-dependent parametrix of this operator, and consider

$$
\wp^{*} \varphi_{0}\left(\begin{array}{cc}
\mathscr{U}^{-1} & 0 \\
0 & 1
\end{array}\right) \mathscr{P}_{Y_{0}}(\lambda)\left(\begin{array}{cc}
\mathscr{U} & 0 \\
0 & 1
\end{array}\right) \wp^{*} \psi_{0}
$$

with the functions $\varphi_{0}, \psi_{0}$ from the proof of Theorem 3.14, This operator belongs

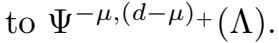

Let $r_{+} P_{0}(\lambda) e_{+}$be a parameter-dependent parametrix of the (interior) pseudodifferential part $r_{+} A_{0}(\lambda) e_{+}$of $A(\lambda)$ according to (3.9), and let

$P(\lambda)=\sum_{Y_{0} \subset Y} \wp^{*} \varphi_{0}\left(\begin{array}{cc}\mathscr{U}^{-1} & 0 \\ 0 & 1\end{array}\right) \mathscr{P}_{Y_{0}}(\lambda)\left(\begin{array}{cc}\mathscr{U} & 0 \\ 0 & 1\end{array}\right) \wp^{*} \psi_{0}+\varphi_{\mathrm{int}}\left(\begin{array}{cc}r_{+} P_{0}(\lambda) e_{+} & 0 \\ 0 & 0\end{array}\right) \psi_{\mathrm{int}}$,

where $\varphi_{\text {int }}$ and $\psi_{\text {int }}$ are as in the proof of Theorem 3.14, By construction, $P(\lambda) \in$ $\Psi^{-\mu,(d-\mu)+(\Lambda)}$ is then a parameter-dependent parametrix of $A(\lambda)$ as desired.

\section{The expansion of the resolvent}

This last section is devoted to the proof of the main result Theorem 1.1. We break it up into two parts, Theorem 4.3 and Theorem 4.4 below. Having the calculus from Section 3 at hand, we are able to argue parallel to the classical arguments for closed manifolds and differential boundary value problems, see [2, 4, 5]. In what follows, we use the notation and conventions from Section 2 .

Let

$$
C_{0}=\gamma_{Y_{0}}\left(\begin{array}{c}
B_{0,1} \\
\vdots \\
B_{0, M_{0}}
\end{array}\right) \mathscr{U} \circ r_{U_{0}}: C^{\infty}(\bar{M}, E) \rightarrow C^{\infty}\left(Y_{0},\left.\bigoplus_{j=1}^{M_{0}} F_{0, j}\right|_{Y_{0} \times\{0\}}\right)
$$

be the coupling condition for $A$ associated with $Y_{0}$ from Definition 2.2, Recall that the operators $B_{0, j}$ have orders $m_{0, j}<m$. For each $j$, choose a family

$$
R_{j}(\lambda): C^{\infty}\left(Y_{0},\left.F_{0, j}\right|_{Y_{0} \times\{0\}}\right) \rightarrow C^{\infty}\left(Y_{0},\left.F_{0, j}\right|_{Y_{0} \times\{0\}}\right)
$$

of order $m-\left(m_{0, j}-1 / 2\right)$ in the calculus of anisotropic parameter-dependent pseudodifferential operators on $Y_{0}$ that is invertible with inverse $R_{j}(\lambda)^{-1}$ being of order $m_{0, j}-1 / 2-m$ in that calculus. Throughout this section, the anisotropy is fixed to be $\ell=m=\operatorname{ord}(A)$.

Let

$$
J_{0,+}=\left.\bigoplus_{j=1}^{M_{0}} F_{0, j}\right|_{Y_{0} \times\{0\}}
$$


and let

$$
T_{0}(\lambda)=\left(\begin{array}{ccc}
R_{1}(\lambda) & & 0 \\
& \ddots & \\
0 & & R_{M_{0}}(\lambda)
\end{array}\right) C_{0}: C^{\infty}(\bar{M}, E) \rightarrow C^{\infty}\left(Y_{0}, J_{0,+}\right) .
$$

Let $T(\lambda)$ be the direct sum of the operators $T_{0}(\lambda)$. Then

$$
A(\lambda)=\left(\begin{array}{c}
A-\lambda \\
T(\lambda)
\end{array}\right): C^{\infty}(\bar{M}, E) \rightarrow \underset{Y_{0} \subset Y}{\bigoplus^{\infty}(\bar{M}, E)} \stackrel{\oplus}{\oplus} C^{\infty}\left(Y_{0}, J_{0,+}\right)
$$

belongs to the operator class $\Psi^{m, m}(\Lambda)$ constructed in Section 3 . The following lemma is immediate.

Lemma 4.2. The boundary contact problem $(A, C)$ is elliptic with parameter in $\Lambda$ in the sense of Definition 2.4 if and only if the operator family $A(\lambda) \in \Psi^{m, m}(\Lambda)$ from (4.1) is parameter-dependent elliptic in the sense of Definition 3.11.

Theorem 4.3. Let $(A, C)$ be elliptic with parameter in $\Lambda$.

a) The operator

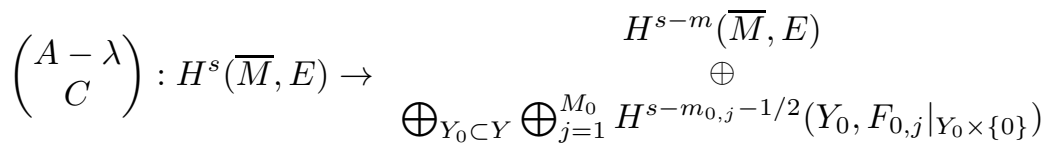

is invertible for all $s>m-1 / 2$ and all $\lambda \in \Lambda$ with $|\lambda|$ sufficiently large.

Consequently, the unbounded operator $A_{C}$ in $L^{2}(\bar{M}, E)$ that acts like $A$ and has domain

$$
\mathcal{D}\left(A_{C}\right)=\left\{u \in H^{m}(\bar{M}, E) ; C u=0\right\}
$$

is closed and densely defined, and for large $\lambda \in \Lambda$ the resolvent $\left(A_{C}-\lambda\right)^{-1}$ exists.

b) There exists $Q(\lambda) \in \Psi^{-m, 0}(\lambda)$ such that $\left(A_{C}-\lambda\right)^{-1}=Q(\lambda)$ for large $\lambda \in \Lambda$. In particular, by Theorem 3.12, the resolvent satisfies the norm estimate

$$
\left\|\left(A_{C}-\lambda\right)^{-1}\right\|_{\mathscr{L}\left(L^{2}(\bar{M}, E)\right)}=O\left(|\lambda|^{-1}\right)
$$

as $|\lambda| \rightarrow \infty$.

Proof. The operator $A(\lambda)$ in (4.1) is parameter-dependent elliptic. Thus, by Theorem 3.17 there exists a parametrix $P(\lambda) \in \Psi^{-m, 0}(\Lambda)$ of $A(\lambda)$. Consequently,

$$
A(\lambda) P(\lambda)-1=R_{1}(\lambda) \in \Psi^{-\infty, 0}(\Lambda) \text { and } P(\lambda) A(\lambda)-1=R_{2}(\lambda) \in \Psi^{-\infty, m}(\Lambda) .
$$

For large $\lambda \in \Lambda$, the operators $1+R_{1}(\lambda)$ and $1+R_{2}(\lambda)$ are invertible as bounded operators in the Sobolev spaces. Write

$$
\left(1+R_{j}(\lambda)\right)^{-1}=1-R_{j}(\lambda)+R_{j}(\lambda) \chi(\lambda)\left(1+R_{j}(\lambda)\right)^{-1} R_{j}(\lambda)
$$

for large $\lambda$, where $\chi$ is a suitable excision function of the origin. From the definition of the class of regularizing operators we obtain that

$$
R_{1}(\lambda) \chi(\lambda)\left(1+R_{1}(\lambda)\right)^{-1} R_{1}(\lambda) \in \Psi^{-\infty, 0}(\Lambda),
$$

and

$$
R_{2}(\lambda) \chi(\lambda)\left(1+R_{2}(\lambda)\right)^{-1} R_{2}(\lambda) \in \Psi^{-\infty, m}(\Lambda) .
$$


Thus $\left(1+R_{j}(\lambda)\right)^{-1}=1+\tilde{R}_{j}(\lambda)$ with regularizing operators $\tilde{R}_{j}(\lambda)$. Consequently,

$$
P(\lambda)\left(1+\tilde{R}_{1}(\lambda)\right)=(Q(\lambda) \quad K(\lambda)) \in \Psi^{-m, 0}(\Lambda)
$$

inverts the operator $A(\lambda)$ for large $\lambda \in \Lambda$. Both a) and b) thus follow in view of

$$
\mathcal{D}\left(A_{C}\right)=\left\{u \in H^{m}(\bar{M}, E) ; T(\lambda) u=0\right\} .
$$

Theorem 4.4. Let $(A, C)$ be elliptic with parameter in the sector $\Lambda$, and let $B \in$ $\operatorname{Diff}^{k}(\bar{M}, E)$. For $N>\frac{\operatorname{dim} \bar{M}+k}{m}$ and large $\lambda \in \Lambda$ the operator

$$
B\left(A_{C}-\lambda\right)^{-N}: L^{2}(\bar{M}, E) \rightarrow L^{2}(\bar{M}, E)
$$

is of trace class, and the trace has an asymptotic expansion

$$
\operatorname{Tr}\left(B\left(A_{C}-\lambda\right)^{-N}\right) \sim|\lambda|^{-N} \sum_{j=0}^{\infty} c_{j}(\hat{\lambda})|\lambda|^{\frac{\operatorname{dim} \bar{M}+k-j}{m}} \text { as }|\lambda| \rightarrow \infty,
$$

where $c_{j}=c_{j}(B, N, A, C) \in C^{\infty}\left(\mathbb{S}^{1} \cap \Lambda\right)$, and $\hat{\lambda}=\lambda /|\lambda|$.

Proof. By Theorem 4.3, we have $\left(A_{C}-\lambda\right)^{-1}=Q(\lambda)$ for large $\lambda \in \Lambda$ with $Q(\lambda) \in \Psi^{-m, 0}(\Lambda)$. Because $B \in \Psi^{k, 0}(\lambda)$, we obtain from the composition theorem (Theorem 3.14) that $B\left(A_{C}-\lambda\right)^{-N}=B Q(\lambda)^{N} \in \Psi^{-N m+k, 0}(\Lambda)$. Since the embed$\operatorname{ding} H^{s}(\bar{M}, E) \hookrightarrow L^{2}(\bar{M}, E)$ is nuclear for $s>\operatorname{dim} \bar{M}$, we get from Theorem 3.12 that

$$
B\left(A_{C}-\lambda\right)^{-N}: L^{2}(\bar{M}, E) \rightarrow H^{N m-k}(\bar{M}, E) \hookrightarrow L^{2}(\bar{M}, E)
$$

is of trace class as an operator in $L^{2}(\bar{M}, E)$ provided that $N m-k>\operatorname{dim} \bar{M}$. This proves the first assertion of the theorem.

In order to show the expansion, it suffices to show that for any $P(\lambda) \in \Psi^{\mu, 0}(\Lambda)$, where $\mu<-\operatorname{dim} \bar{M}$, the $L^{2}$-trace $\operatorname{Tr} P(\lambda)$ has an asymptotic expansion

$$
\operatorname{Tr} P(\lambda) \sim \sum_{j=0}^{\infty} c_{j}(\hat{\lambda})|\lambda|^{\frac{\operatorname{dim} \bar{M}+\mu-j}{m}} \text { as }|\lambda| \rightarrow \infty .
$$

To do this, we decompose $P(\lambda)=r_{+} P_{0}(\lambda) e_{+}+G(\lambda)$ in a pseudodifferential and singular Green operator according to (3.9), and consider the terms separately. The expansion of the trace of the pseudodifferential part $\operatorname{Tr}\left(r_{+} P_{0}(\lambda) e_{+}\right)$follows like in the case of a closed manifold. For the benefit of the reader, we briefly sketch the argument:

Choose a partition of unity $\varphi_{1}, \ldots, \varphi_{M}$ subordinate to a finite covering of $\bar{M}$ by coordinate neighborhoods such that, in addition, $E$ is trivial over each of these neighborhoods. Choose functions $\psi_{j}$ supported in the respective coordinate neighborhoods such that $\psi_{j} \equiv 1$ in a neighborhood of the support of $\varphi_{j}$. Write the operator $P_{0}^{+}(\lambda)=r_{+} P_{0}(\lambda) e_{+}$as

$$
P_{0}^{+}(\lambda)=\sum_{j=0}^{M} \varphi_{j} P_{0}^{+}(\lambda) \psi_{j}+R(\lambda)
$$

where $R(\lambda) \in \Psi^{-\infty, 0}(\Lambda)$. Because $\operatorname{Tr} R(\lambda) \sim 0$, the expansion of $\operatorname{Tr} P_{0}^{+}(\lambda)$ reduces to expanding $\operatorname{Tr}\left(\varphi_{j} P_{0}^{+}(\lambda) \psi_{j}\right)$ for each $j$. This converts to a problem in coordinates. 
In coordinates, the $L^{2}$-trace is given by the integral of the trace of the Schwartz kernel over the diagonal. Thus, if $p(z, \zeta ; \lambda)$ is a local symbol, the trace is given by

$$
(2 \pi)^{-n} \iint \operatorname{tr} p(z, \zeta ; \lambda) d z d \zeta \sim \sum_{j=0}^{\infty}(2 \pi)^{-n} \iint \operatorname{tr} p_{(\mu-j)}(z, \zeta ; \lambda) d z d \zeta \text { as }|\lambda| \rightarrow \infty,
$$

where $p_{(\mu-j)}(z, \zeta ; \lambda)$ is the anisotropic homogeneous component of degree $\mu-j$ of $p, n=\operatorname{dim} \bar{M}$, and $\operatorname{tr}$ denotes the fibrewise trace. Recall that

$$
p_{(\mu-j)}\left(z, \varrho \zeta ; \varrho^{m} \lambda\right)=\varrho^{\mu-j} p_{(\mu-j)}(z, \zeta ; \lambda)
$$

for $\varrho>0$ and $(\zeta, \lambda) \neq(0,0)$. Thus

$$
\begin{aligned}
(2 \pi)^{-n} \iint \operatorname{tr} p_{(\mu-j)} & (z, \zeta ; \lambda) d z d \zeta \\
& =\left((2 \pi)^{-n} \iint \operatorname{tr} p_{(\mu-j)}\left(z,|\lambda|^{-1 / m} \zeta ; \hat{\lambda}\right) d z d \zeta\right) \cdot|\lambda|^{(\mu-j) / m} \\
& =\left((2 \pi)^{-n} \iint \operatorname{tr} p_{(\mu-j)}(z, \zeta ; \hat{\lambda}) d z d \zeta\right) \cdot|\lambda|^{(n+\mu-j) / m} .
\end{aligned}
$$

This shows that the desired asymptotic expansion holds for $\operatorname{Tr} P_{0}^{+}(\lambda)$.

Now consider the trace $\operatorname{Tr} G(\lambda)$. We shall work with the functions $\varphi_{0}$ and $\psi_{0}$ from the proof of Theorem 3.14 (these are not to be confused with the $\varphi_{j}$ 's and $\psi_{j}$ 's above). Write

$$
G(\lambda)=\sum_{Y_{0} \subset Y} \varphi_{0} G(\lambda) \psi_{0}+\tilde{G}(\lambda)
$$

with $\tilde{G}(\lambda) \in \Psi^{-\infty, 0}(\Lambda)$. In view of $\operatorname{Tr} \tilde{G}(\lambda) \sim 0$, we only need to expand the trace $\operatorname{Tr}\left(\varphi_{0} G(\lambda) \psi_{0}\right)$ for each $Y_{0}$. From the trace property we get

$$
\operatorname{Tr}\left(\varphi_{0} G(\lambda) \psi_{0}\right)=\operatorname{Tr}\left(\mathscr{U} \circ\left(\varphi_{0} G(\lambda) \psi_{0}\right) \circ \mathscr{U}^{-1}\right)
$$

with the canonical map $\mathscr{U}$ from (2.1). The operator $G_{Y_{0}}(\lambda)=\mathscr{U} \circ\left(\varphi_{0} G(\lambda) \psi_{0}\right) \circ$ $\mathscr{U}^{-1}$ is an anisotropic parameter-dependent singular Green operator of order $\mu$ and type zero in Boutet de Monvel's calculus on $Y_{0} \times[0, \varepsilon)$ supported near the boundary. Hence we know that

$$
\operatorname{Tr} G_{Y_{0}}(\lambda) \sim \sum_{j=0}^{\infty} d_{j}(\hat{\lambda})|\lambda|^{\frac{(n-1)+\mu-j}{m}} \text { as }|\lambda| \rightarrow \infty .
$$

For the benefit of the reader, let us stress that the latter expansion is proved following the same scheme as the expansion of the pseudodifferential part above:

A similar localization argument as the one given above reduces the task of expanding $\operatorname{Tr} G_{Y_{0}}(\lambda)$ to expanding the trace in coordinates near the boundary. The boundary symbols $g(y, \eta ; \lambda)$ of $G_{Y_{0}}(\lambda)$ have the structure explained in Remark 3.5 In coordinates, the $L^{2}$-trace is given by

$$
(2 \pi)^{-(n-1)} \iint \operatorname{tr} g(y, \eta ; \lambda) d y d \eta,
$$

where tr denotes the trace on the space $L^{2}\left(\overline{\mathbb{R}}_{+}\right) \otimes \mathbb{C}^{\left.\operatorname{dim} \wp ! E\right|_{Y_{0}}}$. As noted in Remark 3.5. $\operatorname{tr} g(y, \eta ; \lambda)$ is an ordinary parameter-dependent symbol of order $\mu$. The homogeneous components are the $\operatorname{tr} g_{(\mu-j)}(y, \eta ; \lambda)$, where, for each $j, g_{(\mu-j)}(y, \eta ; \lambda)$ is the (twisted) homogeneous component of degree $\mu-j$ associated with $g(y, \eta ; \lambda)$. Thus the same argument as above implies the expansion of the trace as desired. 


\section{References}

[1] D. Freed and R. Melrose, A mod $k$ index theorem, Invent. Math. 107 (1992), no. 2, 283-299.

[2] P. Gilkey, Invariance theory, the heat equation, and the Atiyah-Singer index theorem, 2nd ed., Studies in Advanced Mathematics, CRC Press, Boca Raton, 1995.

[3] _ Asymptotic formulae in spectral geometry, Studies in Advanced Mathematics, Chapman \& Hall/CRC, Boca Raton, 2004.

[4] G. Grubb, Functional calculus of pseudodifferential boundary problems, 2nd ed., Progress in Mathematics, vol. 65. Birkhäuser, Basel, 1996.

[5] — A weakly polyhomogeneous calculus for pseudodifferential boundary problems, J. Funct. Anal. 184 (2001), 19-76.

[6] V. Kostrykin and R. Schrader, Laplacians on metric graphs: Eigenvalues, resolvents, and semigroups, in G. Berkolaiko, R. Carlson, S.A. Fulling, and P. Kuchment (Eds.), Quantum Graphs and Their Applications, Contemp. Math. Vol. 415, Amer. Math. Soc., Providence, RI, 2006, pp. 201-225.

[7] P. Kuchment, Quantum graphs I. Some basic structures, Waves Random Media 14 (2004), S107-S128.

[8] M. Lesch, Operators of Fuchs type, conical singularities, and asymptotic methods, Teubner Texte zur Mathematik, vol. 136, Teubner-Verlag, Leipzig, 1997.

[9] J. Rosenberg, Groupoid $C^{*}$-algebras and index theory on manifolds with singularities, Geom. Dedicata 100 (2003), 65-84.

[10] A. Savin and B. Sternin, The index defect in the theory of nonlocal problems and the $\eta$ invariant, Sb. Math. 195 (2004), no. 9-10, 1321-1358.

[11] E. Schrohe, A short introduction to Boutet de Monvel's calculus, Oper. Theory Adv. Appl. 125 (2001), 85-116.

[12] B.-W. Schulze, Boundary value problems and singular pseudo-differential operators, Pure and Applied Mathematics. John Wiley \& Sons, Ltd., Chichester, 1998.

[13] R. Seeley, Complex powers of an elliptic operator, Singular Integrals (Proc. Sympos. Pure Math., Chicago, Ill., 1966), pp. 288-307, Amer. Math. Soc., 1967.

[14] - The resolvent of an elliptic boundary problem, Amer. J. Math. 91 (1969), 889-920.

[15] M. Shubin, Pseudodifferential operators and spectral theory, Springer Verlag, Princeton, N.J., 1987.

Penn State Altoona, 3000 Ivyside Park, Altoona, PA 16601, U.S.A.

E-mail address: krainer@psu.edu 